\title{
Bulk-Density Representations of Branched Planar Ice Crystals: Errors in the Polarimetric Radar Variables
}

\author{
Robert S. SCHROM AND MATTHEW R. KUMJIAN \\ Department of Meteorology and Atmospheric Science, The Pennsylvania State University, University Park, Pennsylvania
}

(Manuscript received 25 April 2017, in final form 11 October 2017)

\begin{abstract}
Recent interest in interpreting polarimetric radar observations of ice and evaluating microphysical model output with these observations has highlighted the importance of accurately computing the scattering of microwave radiation by branched planar ice crystals. These particles are often represented as spheroids with uniform bulk density, reduced from that of solid ice to account for the complex, nonuniform structure of natural branched crystals. In this study, the potential errors that arise from this assumption are examined by comparing scattering calculations of branched planar crystals with those of homogeneous, reduced-density plate crystals and spheroids with the same mass, aspect ratio, and maximum dimension. The results show that this assumption leads to significant errors in backscatter cross sections at horizontal and vertical polarization, specific differential phase $\left(K_{\mathrm{DP}}\right)$, and differential reflectivity $\left(Z_{\mathrm{DR}}\right)$, with the largest $Z_{\mathrm{DR}}$ errors for ice crystals with the most extreme aspect ratios $(<0.01)$ and effective densities $<250 \mathrm{~kg} \mathrm{~m}^{-3}$. For example, the maximum errors in X-band $Z_{\mathrm{DR}}$ are $4.5 \mathrm{~dB}$ for 5.6-mm branched planar crystals. However, substantial errors are present at all weather radar frequencies, with resonance scattering effects at $\mathrm{Ka}$ and $\mathrm{W}$ band amplifying the lowfrequency errors. The implications of these results on the interpretation of polarimetric radar observations and forward modeling of the polarimetric radar variables from microphysical model output are discussed.
\end{abstract}

\section{Introduction}

One of the challenges in using polarimetric radar to study ice microphysical processes is determining an appropriate forward model to map conceptual or microphysical models of ice particles to the radar observables. An early study by Hall et al. (1984) examines S-band polarimetric radar measurements of precipitation and explains observations of positive differential reflectivity $\left(Z_{\mathrm{DR}}\right)$ in ice using scattering calculations for oblate spheroids with a homogeneous bulk density below that of solid ice. This reduced density approximates the complex structure of pristine ice crystals and aggregates as a random distribution of mass within the particle's bounding region. Therefore, in scattering calculations, ice particles with aspect ratios $<1$, such as platelike crystals and aggregates, are modeled as oblate spheroids, and ice particles with aspect ratios $>1$, such as columns and conical graupel, are modeled as prolate spheroids. Aspect ratio defined herein is the ratio of the ice crystal $c$ axis (along the prism face) to the ice crystal $a$ axis (along the basal face). The main advantage in

Corresponding author: Robert S. Schrom, rss5116@psu.edu using these shapes is that their scattering can be calculated analytically for particles small relative to the wavelength (Bohren and Huffman 1983).

Many subsequent studies using polarimetric radar observations of ice particles at wavelengths between $\mathrm{S}$ and $\mathrm{Ka}$ band have also modeled their scattering with homogeneous, reduced-density spheroids (e.g., Vivekanandan et al. 1994; Matrosov et al. 1996; Ryzhkov et al. 1998; Spek et al. 2008; Kennedy and Rutledge 2011; Andrić et al. 2013; Bechini et al. 2013; Thompson et al. 2014; Moisseev et al. 2015). The use of this simplified scattering model has allowed for some qualitative microphysical interpretations of these observations. In particular, the existence of locally enhanced $Z_{\mathrm{DR}}$ and specific differential phase $\left(K_{\mathrm{DP}}\right)$ near $-15^{\circ} \mathrm{C}$ has been linked to the presence of oblate ice particles growing through vapor deposition; $Z_{\mathrm{DR}}$ and $K_{\mathrm{DP}}$ subsequently decrease as aggregation and/or riming dominate growth (e.g., Kennedy and Rutledge 2011; Andrić et al. 2013). However, quantitative information about vapor deposition, aggregation, and riming magnitudes, as well as information about the secondary ice particle habits responsible for this signature, has remained elusive.

Recent advancements in ice microphysical modeling may provide additional constraints on some of this 
uncertain information. In particular, schemes developed by Hashino and Tripoli (2007), Harrington et al. (2013), Jensen and Harrington (2015), and Morrison and Milbrandt (2015) have sought to reduce errors in using fixed ice particle classes by allowing particles to grow with naturally evolving sizes, aspect ratios, and/or bulk densities during vapor deposition, aggregation, and riming. The potential to evaluate these models with polarimetric radar observations has prompted interest in the development of forward models that accurately transform model output to polarimetric radar observables (e.g., Ryzhkov et al. 2011; Sulia and Kumjian 2017). Given the rapid growth of branched planar crystals during vapor deposition (e.g., Fukuta and Takahashi 1999) and efficient growth through aggregation as these particles self-collect (e.g., Connolly et al. 2012), these particles can have large impacts on precipitation growth above the melting layer. Additionally, evaluating these processes in microphysical models with polarimetric radar observations requires an accurate radar forward model for branched planar crystals. It is therefore important to assess errors using homogeneous, reduced-density particles to compute the scattering of complex-structured branched planar ice crystals.

Several recent studies suggest that using bulk density to characterize the complex structure of ice particles may introduce significant error in the simulated radar variables. Botta et al. (2011) and Kneifel et al. (2015) show that representing aggregates as homogeneous, bulkdensity spheroids introduces errors in the backscatter cross section at vertical incidence, especially as the particle size approaches the scale of the radar wavelength. For branched planar crystals, Botta et al. (2013) show a large variability in backscatter cross section at $\mathrm{W}$ band from 5-mm crystals with a range of different structures.

Based solely on these results, resonance effects would seem to play the dominant role in discrepancies in the backscatter cross sections between branched planar crystals and their homogeneous plate representations. Furthermore, this implies that accurately representing the ice particle structure in scattering calculations is only necessary for particles with sizes on the order the radar wavelength and larger. Indeed, numerous applications of this hypothesis, with limited justification, are found in the literature. For example, Vivekanandan et al. (1994, p. 2) state, "In the frequency region between $S$ and $\mathrm{K}$ band $(3-35 \mathrm{GHz})$, the detailed structure of ice crystal shapes is less important if the sizes of the scatterers are small compared to the wavelength," and Thompson et al. (2014, p. 1459) similarly justify their use of homogeneous spheroids as follows: "All hydrometeors are modeled as oblate spheroids without branched or otherwise irregular shapes, which is sufficient for X-, C-, and S-band weather radar applications."
Westbrook (2014) briefly addresses the validity of this assumption with scattering calculations of branched planar crystals of a single aspect ratio (0.1) at $\mathrm{S}$ band. Calculations for homogeneous plates with the same mass and dimensions (and thus reduced bulk density) show close correspondence to calculations for branched planar crystals. However, natural branched planar crystals often have much lower aspect ratios and more complicated internal structures than the particles used in Westbrook (2014) (e.g., Auer and Veal 1970; Bailey and Hallett 2009).

The purpose of this study is therefore to expand on the results from Westbrook (2014) and assess the differences between the long-wavelength polarimetric signatures of branched planar crystals and homogeneous, reduced-density plates for the large variety of branched planar crystals contained in the Pennsylvania State University (PSU) scattering database (Lu et al. 2016). We use homogeneous, reduced-density plates rather than homogeneous, reduced-density spheroids in our comparisons to directly test the validity of the homogeneous, reduced-density assumption and avoid additional errors in representing the scattering of hexagonal particles with spheroids (e.g., Westbrook 2014). We briefly show for $Z_{\mathrm{DR}}$ that this source of error is relatively small compared with the assumption of homogeneity. We further assess the errors in using homogeneous plates to represent the scattering of two branched planar crystals at shorter wavelengths where resonance effects may be significant.

To illustrate the theoretical impacts of ice crystal structure on the radar variables, we present an overview of ice particle scattering in section 2. In section 3, we discuss the branched planar crystals contained in the PSU scattering database and present our method for generating homogeneous, reduced-density plates from the properties of particles in the database. A comparison of the radar variables for homogeneous plates and branched planar crystals at $\mathrm{X}$ band, physical explanations of these results using internal field calculations, and similar comparisons for the two branched planar crystals at multiple wavelengths are shown in section 4 . Finally, we discuss the implications of these results and our conclusions in section 5 .

\section{Ice crystal scattering}

Following Bohren and Huffman (1983), we consider ice crystals to be composed of small subregions that scatter as oscillating electric dipoles. At X, C, and S band, pristine ice particles are generally much smaller than the radar wavelength and are often referred to as scattering in the Rayleigh or small-particle regime. In this regime, we may consider the incident wave inside 
the particle to be spatially homogeneous, and thus dipoles within the particle oscillate in phase (van de Hulst 1981). The incident wave aligns dipoles in the polarization direction (in a manner described by the dielectric function), and these dipoles each produce electric fields with the same orientation. The electric field around each dipole may also strengthen or weaken the electric fields of neighboring dipoles; these secondary responses of the neighboring dipoles are known as near-field interactions (e.g., Lu et al. 2013). Dipoles positioned along the direction of polarization enhance each other's electric fields; dipoles positioned normal to the direction of polarization weaken each other's electric fields. The near-field component of the electric field around each dipole changes intensity with distance $r$ by a factor of $r^{-3}$ (Jackson 1975), and therefore closely packed dipoles (i.e., densely distributed mass) lead to stronger near-field interactions. As a result, a horizontally positioned (e.g., along the $x$ direction) array of single dipoles illuminated by a horizontally polarized incident field will have a stronger electric field than the same array of dipoles illuminated by a vertically polarized (along the $z$ direction) incident field (e.g., Lu et al. 2014).

The near-field interactions between dipoles within an ice particle produce net dipole moments that depend on the incident wave polarization; these dipole moments are related to the total enhancement or reduction of the particle's internal electric field intensity. From these net dipole moments, we can derive the radar variables. The dipole moment at a given polarization is proportional to the scattering amplitude at that polarization (Jackson 1975); these scattering amplitudes are used directly to calculate the polarimetric radar variables (e.g., Ryzhkov et al. 2011).

We first examine the backscatter cross section $(\sigma)$, the product of the efficiency with which power is backscattered by a particle and the cross-sectional area of the particle, defined as (Doviak and Zrnić 1993)

$$
\sigma_{h, v}=4 \pi\left|S_{h h, v v}\right|^{2},
$$

where subscripts represent the polarization (either horizontal $h$ or vertical $v$ ), $S_{h h, v v}$ has units of millimeters and is the scattering amplitude in the backscatter direction, and its subscript indicates that the polarization (either horizontal or vertical) is the same for the incident and scattering directions.

The $Z_{\mathrm{DR}}$, related to the difference in backscattered power at horizontal and vertical polarization, is defined for a single particle as (Doviak and Zrnić 1993)

$$
Z_{\mathrm{DR}}=10 \times \log _{10}\left(\frac{\sigma_{h}}{\sigma_{v}}\right),
$$

where positive values are thus associated with particles that have greater dipole moments for horizontally polarized incident radiation than for vertically polarized incident radiation. As discussed above, the shape of the particle (i.e., the alignment and distribution of dipoles) will impact the relative enhancement or reduction of the electric field at each polarization because of near-field interactions, and therefore will impact the particle's $Z_{\mathrm{DR}}$.

At wavelengths on the order of the particle size, the scattered field multipole terms become important (Jackson 1975). These reflect the fact that individual dipoles within the particle no longer oscillate in phase. Because the total scattered field depends on how the scattered waves from individual dipoles add together, this incoherent superposition (referred to herein as resonance effects) leads to $\sigma_{h}$ and $\sigma_{v}$ values that do not necessarily increase monotonically with particle mass, as $\sigma_{h}$ and $\sigma_{v}$ do for particles small relative to the wavelength.

\section{Scattering calculations}

\section{a. Ice crystal scattering database}

To better interpret radar and satellite observations, Lu et al. (2016) present a database (Aydin et al. 2016) containing scattering calculations for realistic ice particle shapes. The database includes aggregates, branched planar crystals, plates, columns, and conical graupel; we focus on the calculations for branched planar crystals.

All of the branched planar crystals in the database are bounded by a hexagonal prism characterized by a thickness $(h)$ and maximum horizontal dimension $(d)$. For a given $d, h$ is assigned using the empirical fit of branched planar crystals dimensions observed by Auer and Veal (1970). The branched planar crystals in the database have values of $d$ between 0.5 and $5.6 \mathrm{~mm}$; for each of these values, the particle has realizations with two different $h: h$ given by Auer and Veal (1970), and one-half that value to capture additional spread in aspect ratio (referred to herein as 1.0- and 0.5-thicknessfactor particles, respectively). Because the database particles comprise discrete numbers of finite-volume dipoles, the true aspect ratios of the database particles are slightly different from the values given by Auer and Veal (1970). However, because this error is small $(<0.25 \%)$ and minor relative to the spread in aspect ratios from Auer and Veal (1970), it will have a minimal impact on the scattering results.

For each particle of a given $d$ and $h$, the database contains several realizations of branched planar crystal structure. These realizations use a range of dimensions for the core and branches, and a range of locations, numbers, and dimensions for the subbranches to capture the variability of natural ice crystals. Because of the 
greater degrees of freedom in the arrangement of these components in large ice crystals, the number of realizations and variability in mass generally increases as $d$ increases. The scattering amplitudes of all of the resulting particles are then calculated using the discrete dipole approximation (DDA; Draine and Flatau 1994).

\section{b. Homogeneous, reduced-density plates}

For each branched planar crystal in the database, we create a homogeneous plate with the same $d, h$, and mass, thus requiring us to assign the homogeneous plate a reduced effective density. Because the branched planar crystals in the database vary only in the plane containing their $a$ axes, we use the ratio of the particle area projected onto this plane to the area of the hexagon bounding the particle to calculate the effective density with

$$
\rho_{\mathrm{hp}}=\frac{8 A_{\mathrm{db}}}{3 \sqrt{3} d^{2}} \rho_{i}
$$

where $A$ is the area projected onto the plane containing the $a$ axis; the subscripts hp and db represent homogeneous plate and corresponding branched planar database crystal, respectively; and $\rho_{i}$ is the density of solid ice. We next need to map this effective density $\rho_{\text {hp }}$ to a dielectric constant that captures the response to electromagnetic radiation of uniformly distributed solid-ice dipoles in a medium of air.

Many previous works have examined methods for representing the scattering of an effective medium with two components: a surrounding medium known as the matrix, and discrete particles of a given material known as the inclusions (e.g., Maxwell Garnett 1904; Shivola 1989). The simplest and most widely used method for quantifying the dielectric constant of this effective medium is to use the Maxwell Garnett model, in which the inclusions are spheres. More complex effective medium models use ellipsoids, where their size, shape, and orientation may be described by a probability distribution (e.g., Bohren and Huffman 1983). Westbrook (2014) uses randomly oriented rods as the inclusions, suggesting that these shapes better approximate the branches and subbranches of dendrites. However, these inclusions are still somewhat unphysical and may not be the most accurate for other ice particles (e.g., partially rimed pristine ice and bullet rosettes). Thus, we proceed using the Maxwell Garnett mixing formula (Maxwell Garnett 1904) as given by (Ryzhkov et al. 2011)

$$
\varepsilon_{\mathrm{hp}}=\frac{1+2 \frac{\rho_{\mathrm{hp}}}{\rho_{i}} \frac{\varepsilon_{i}-1}{\varepsilon_{i}+2}}{1-\frac{\rho_{\mathrm{hp}}}{\rho_{i}} \frac{\varepsilon_{i}-1}{\varepsilon_{i}+2}},
$$

where $\varepsilon_{\mathrm{hp}}$ is the reduced dielectric constant for the homogeneous plate and $\varepsilon_{i}$ is the dielectric constant for solid ice. The above formula is valid for spherical ice inclusions in an air matrix. We choose ice as the inclusions and air as the matrix because this effective medium is most appropriate for the sparse dendrites; given that sparse dendrites have the lowest effective density, these particles should provide the strongest test of the validity of the homogeneous particle representation idea. In addition, the effective dielectric constant resulting from using air inclusions in an ice matrix converges to the effective dielectric constant from ice inclusions in an air matrix as the density approaches solid ice.

We then create solid hexagonal plates composed of a lattice of dipoles, each with reduced dielectric constant $\varepsilon_{\text {hp }}$ and compute the scattering of these particles with the ADDA code of Yurkin and Hoekstra (2011). This procedure is analogous to that used for homogeneous, reduced-density spheroids in previous studies. All calculations used in the comparisons that follow are performed at side incidence with no canting and are valid at $\mathrm{X}$ band unless otherwise noted. Because our calculations and previous works (e.g., Lemke and Quante 1999) show negligible dependence on the azimuthal rotation of small particles within the horizontal plane (i.e., at side incidence), all of our results are for particles with the same fixed azimuthal orientation.

\section{Comparison of simulated radar variables}

\section{a. Ice particles small relative to the wavelength}

To illustrate differences in near-field interactions within branched planar crystals and the corresponding homogeneous plates, we examine errors in $\sigma_{h}, \sigma_{v}, Z_{\mathrm{DR}}$, and $K_{\mathrm{DP}}$. We define these errors as the difference between the quantity for the homogeneous plate and the quantity for the corresponding database branched planar crystal used to generate the homogeneous plate. Values of $\sigma_{h}, \sigma_{v}$, and their errors are given in decibels $\left[10 \times \log 10\left(\mathrm{~mm}^{2}\right)\right]$ for convenience.

An evaluation of $\sigma_{h}$ error is shown in Fig. 1. The homogeneous plates underestimate $\sigma_{h}$ by $1-3.5 \mathrm{~dB}$, with the errors becoming more negative with decreasing $\rho_{\text {hp }}$. The 0.5 -thickness-factor particles generally have more negative errors relative to the 1.0-thickness-factor particles for a given $\rho_{\mathrm{hp}}$.

A slight majority of the homogeneous plate calculations underestimate $\sigma_{v}$, though by $<1 \mathrm{~dB}$ (Fig. 2). However, in contrast to the results for $\sigma_{h}$, the lowest$\rho_{\mathrm{hp}}$ plates overestimate $\sigma_{v}$, by as much as $1.5 \mathrm{~dB}$ for the 0.5 -thickness-factor particles with $\rho_{\mathrm{hp}}<250 \mathrm{~kg} \mathrm{~m}^{-3}$. The $\sigma_{v}$ differences between the homogeneous plates and branched planar crystals are generally larger 


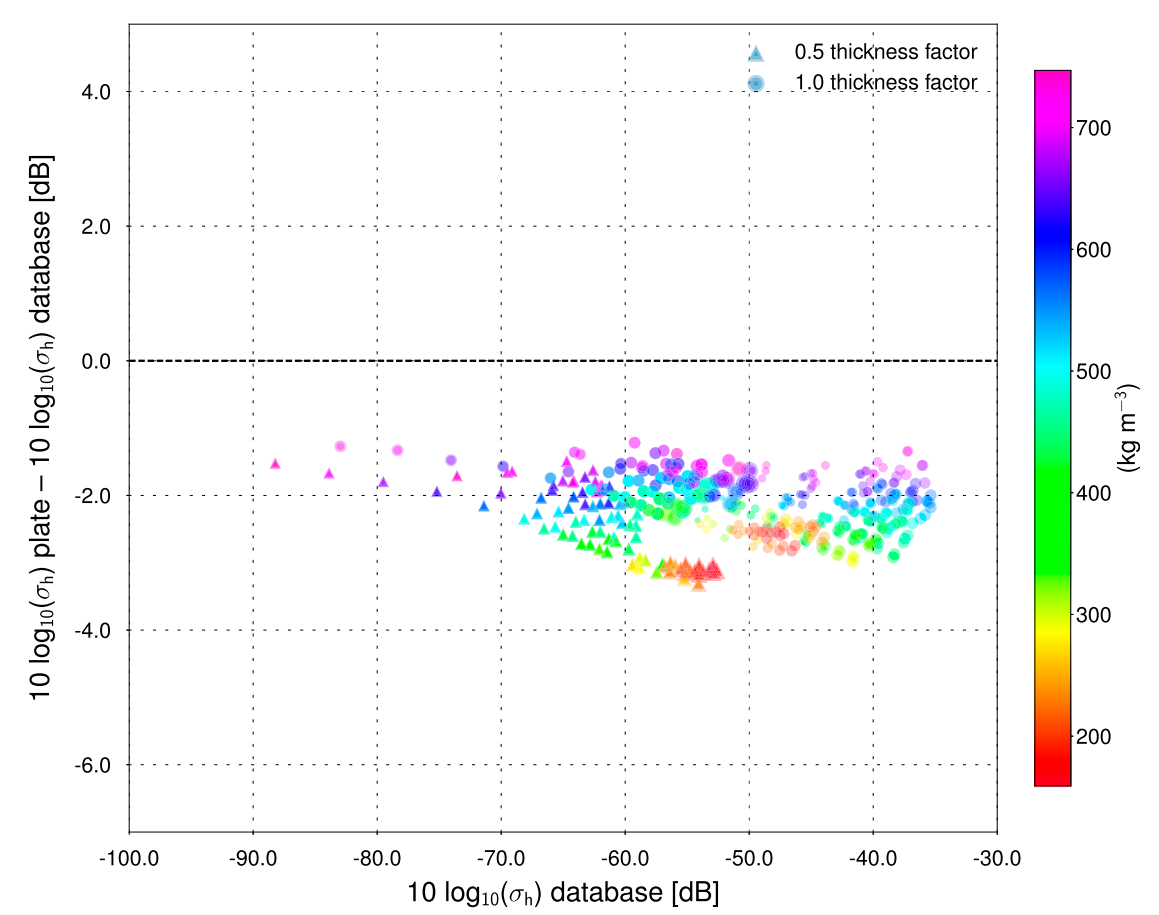

FIG. 1. Scatterplot of the difference in $\sigma_{h}$ between the branched planar crystals and the corresponding homogeneous reduced-density plates vs the $\sigma_{h}$ of the branched planar crystals; the scattering of the branched planar crystals comes from the Lu et al. (2016) database. The color of each marker indicates the $\rho_{\mathrm{hp}}$ of the particles and the size of each marker is proportional to the inverse of aspect ratio (larger markers indicate lower aspect ratio). Triangular markers indicate particles with a factor of 0.5 applied to the thickness-maximum dimension relationship from Auer and Veal (1970) and circular markers indicate that a factor of 1.0 is applied. The zero-error line is indicated by the dashed black contour.

for the 0.5-thickness-factor particles than for the 1.0-thickness-factor particles.

As observed in the $\sigma_{h}$ comparisons, there is a clear relationship between $\rho_{\mathrm{hp}}$ and $\sigma_{v}$ error. As $\rho_{\mathrm{hp}}$ decreases, the difference between the plate $\sigma_{v}$ and the branched planar $\sigma_{v}$ increases. At horizontal polarization, increasingly sparse dendrites maintain strong near-field enhancements relative to those of equivalent, reduceddensity homogeneous plates because of the tightly packed dipoles in the branches and subbranches of these particles. For vertically polarized radiation, these dipoles packed closely in the horizontal plane lead to strong reductions in the internal field magnitudes relative to those in the equivalent, reduced-density homogeneous plate. A further exploration of the near-field interactions is provided in the following subsection.

Differences in $Z_{\mathrm{DR}}$ between the homogeneous plates and the branched planar crystals reveal the combined effects of the errors in the $\sigma_{h}$ and $\sigma_{v}$ results. The results for $Z_{\mathrm{DR}}$ (Fig. 3) show that all the homogeneous plates underestimate the $Z_{\mathrm{DR}}$ of the corresponding branched planar crystals, with the most substantial errors of $\sim-4.5 \mathrm{~dB}$ occurring for 0.5 -thickness-factor particles with $\rho_{\mathrm{hp}}<250 \mathrm{~kg} \mathrm{~m}^{-3}$. For a given branched planar $Z_{\mathrm{DR}}$, there is a clear relationship between $\rho_{\mathrm{hp}}$ and the error: as $\rho_{\mathrm{hp}}$ increases, the magnitude of the $Z_{\mathrm{DR}}$ error decreases.

We observe some clustering of the 1.0-thickness-factor particles into two groups by values of branched planar $Z_{\mathrm{DR}}$ and $Z_{\mathrm{DR}}$ error (Fig. 3). The first cluster has $Z_{\mathrm{DR}}$ errors $>-3.3 \mathrm{~dB}$ and branched planar $Z_{\mathrm{DR}}<6 \mathrm{~dB}$ for the lowest- $\rho_{\mathrm{hp}}$ particles, with $Z_{\mathrm{DR}}$ errors and branched planar $Z_{\mathrm{DR}}$ values increasing with $\rho_{\mathrm{hp}}$ to $\sim-0.2$ and $\sim 7 \mathrm{~dB}$, respectively. A second cluster of 1.0-thickness-factor particles generally corresponds to the $Z_{\mathrm{DR}}$ error and branched planar $Z_{\mathrm{DR}}$ values of the 0.5 -thickness-factor particles with $\rho_{\mathrm{hp}}>300 \mathrm{~kg} \mathrm{~m}^{-3}$. The most substantial $Z_{\mathrm{DR}}$ errors in this cluster are $\sim-3.9 \mathrm{~dB}$ and increase to $\sim-0.4 \mathrm{~dB}$ for the highest- $\rho_{\mathrm{hp}}$ particles. These clusters are likely a complex function of the relative dimensions and shapes of the cores, subbranches, and branches of the database particles. These results suggest that we need additional quantities describing these components of branched planar crystals to fully characterize their intrinsic polarimetric radar observables. 


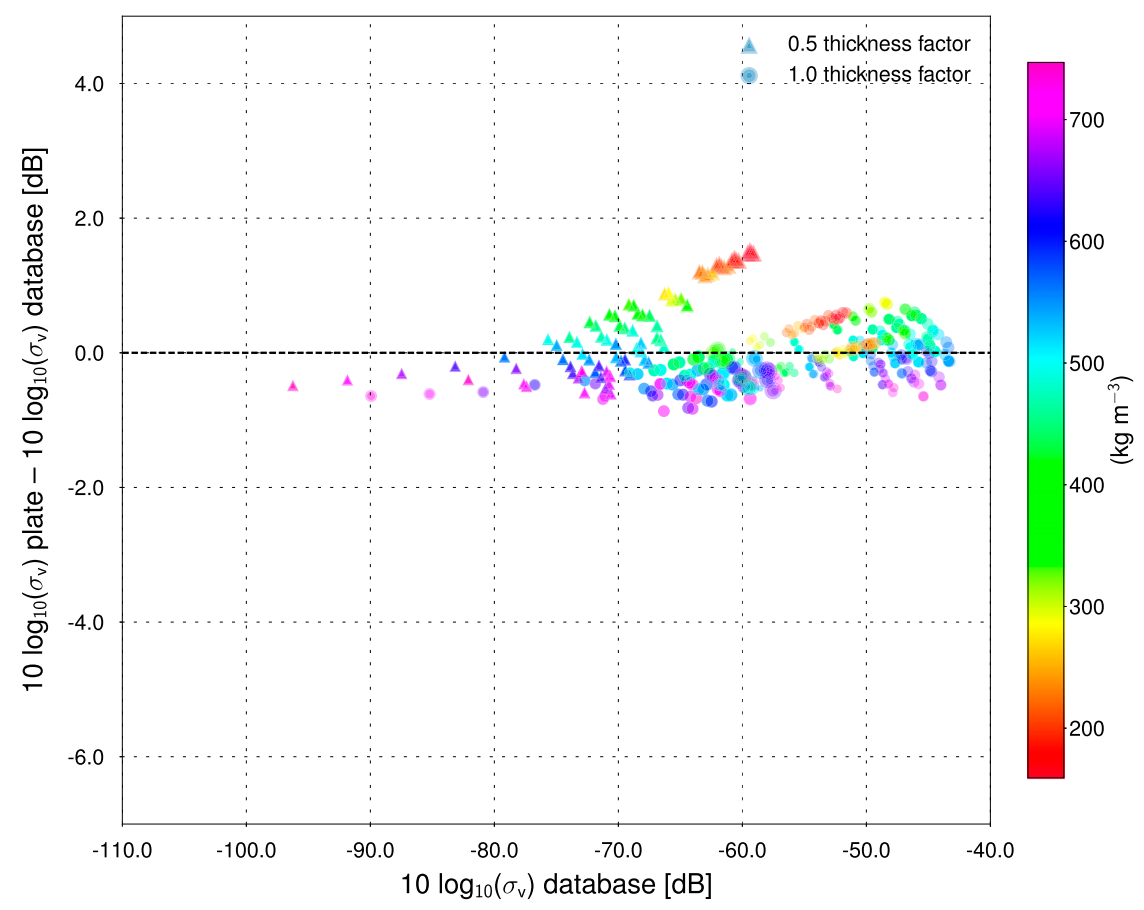

FIG. 2. As in Fig. 1, but for $\sigma_{v}$.

Given that Westbrook (2014) shows minor differences between the polarizabilities of 0.1 -aspect-ratio branched planar crystals and corresponding reduceddensity homogeneous plates, we show that these results are consistent with our results for branched planar crystals with more realistic aspect ratios. Because the calculations from Westbrook (2014) for stellar and dendritic crystals are similar, we reproduce only the more simplistic-shaped stellar crystal calculations with DDA. As in Westbrook (2014), the particles have a range of ice volume fractions from 0 to 1 and a fixed aspect ratio of 0.1 .

The $Z_{\mathrm{DR}}$ calculations for these particles (Fig. 3) show the $0-\mathrm{dB}$ error for the solid-ice plate decreasing to a minimum value of $\sim-1 \mathrm{~dB}$ for the particle with effective density $\sim 500 \mathrm{~kg} \mathrm{~m}^{-3}$, before increasing back toward $0 \mathrm{~dB}$ as the effective density continues to decreases. This behavior is consistent with our results for the branched planar crystals in the database, where the errors increase toward $0 \mathrm{~dB}$ as the effective density increases. However, we do not observe any set of branched planar crystals where the $Z_{\mathrm{DR}}$ errors become less negative as the effective density decreases. If lower-effective-density branched planar crystals with comparable aspect ratios dictated by the Auer and Veal (1970) data were shown, the $Z_{\mathrm{DR}}$ error would eventually become less negative with decreasing effective density. Based on our results, the effective density where the $Z_{\mathrm{DR}}$ would begin to become less negative is $<170 \mathrm{~kg} \mathrm{~m}^{-3}$. As the aspect ratio increases toward 1 , the effective density of the most negative error increases, as evident in the stellar crystal $Z_{\mathrm{DR}}$ results.

One of the additional conclusions noted by Westbrook (2014) is that solid-ice spheroids of the same aspect ratio as solid-ice plates overestimate $Z_{\mathrm{DR}}$ by as much as $1.6 \mathrm{~dB}$. This maximum $Z_{\mathrm{DR}}$ error occurs at an aspect ratio of $1 / 3$, decreasing to $0.2 \mathrm{~dB}$ as the aspect ratio reaches $1 / 100$. Because homogeneous reduced-density spheroids are frequently used to represent branched planar crystals (e.g., Kennedy and Rutledge 2011; Andrić et al. 2013; Sulia and Kumjian 2017), we briefly explore whether the $Z_{\mathrm{DR}}$ errors observed for the homogeneous plates are similar to the errors in using homogeneous spheroids, where $Z_{\mathrm{DR}}$ is higher.

A scatterplot of the $Z_{\mathrm{DR}}$ error for spheroids versus the $Z_{\mathrm{DR}}$ error for plates (Fig. 4) shows that the differences between the two shapes are minimal for the lowest-density particles that have the most substantial $Z_{\mathrm{DR}}$ errors $(<-4 \mathrm{~dB})$. As the effective density increases, the difference in $Z_{\mathrm{DR}}$ error between the spheroids and plates increases; the highest-density $\left(>650 \mathrm{~kg} \mathrm{~m}^{-3}\right)$ particles have spheroid $Z_{\mathrm{DR}}$ errors $0.5 \mathrm{~dB}$ greater than the plates. These results imply that as the ice crystals become sparser, the $Z_{\mathrm{DR}}$ errors from underestimating the near-field interactions outweigh the overestimates in $Z_{\mathrm{DR}}$ from using spheroids to represent hexagonal prism-based shapes.

Last, comparisons of $K_{\mathrm{DP}}$ between the branched planar crystals and the corresponding homogeneous plates show that, similar to the analysis of $Z_{\mathrm{DR}}$, the 


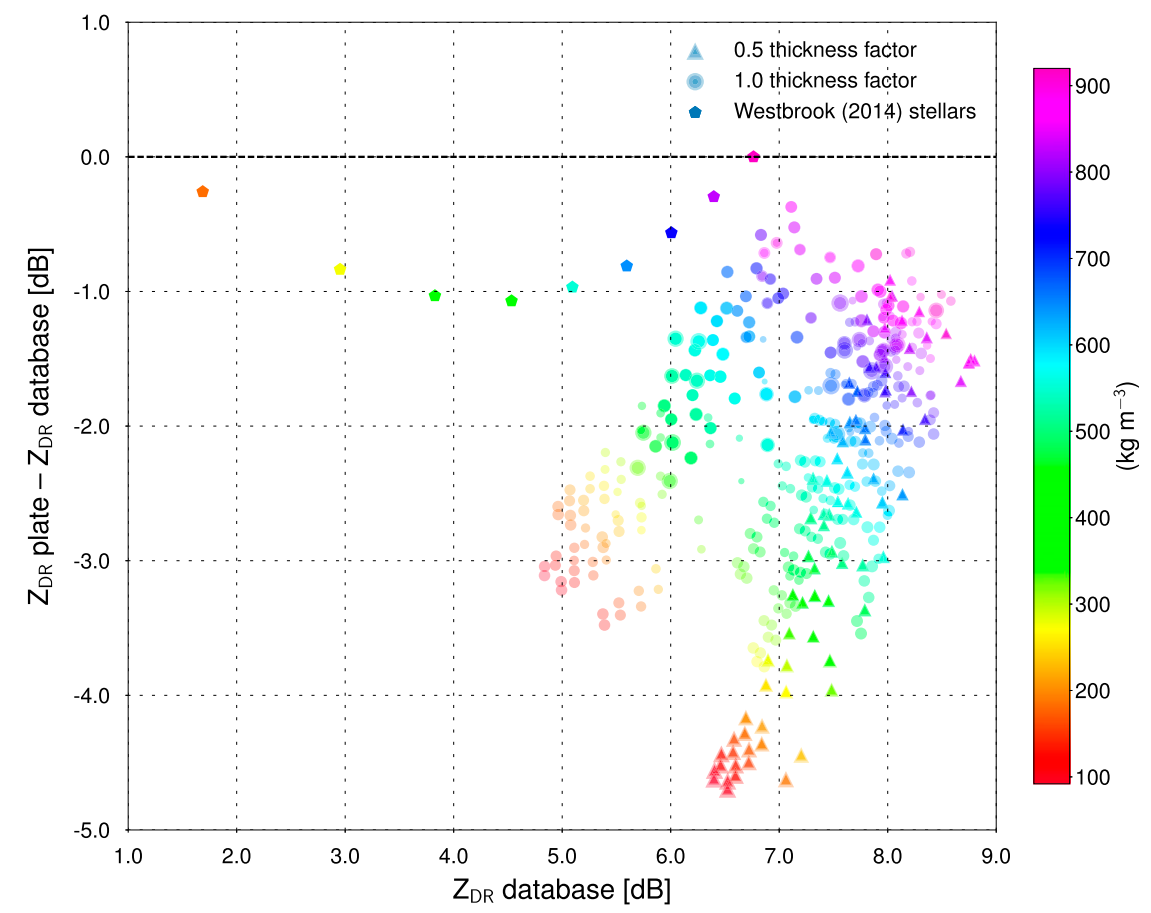

FIG. 3. As in Fig. 1, but for $Z_{\mathrm{DR}}$. The size of the markers indicating the Westbrook (2014) particles are enlarged for the purposes of interpretation and therefore do not correspond in scale to the size of the markers depicting the Lu et al. (2016) branched planar crystals.

homogeneous plates underestimate the values, with the errors increasing in magnitude as effective density decreases (Fig. 5). The equation for $K_{\mathrm{DP}}$ is given in Bringi and Chandrasekar (2001) as

$$
K_{\mathrm{DP}}=\frac{0.180 \lambda}{\pi} n \operatorname{Re}\left(S_{h h}-S_{v v}\right),
$$

where $K_{\mathrm{DP}}$ is in degrees per kilometer, $\lambda$ is the radar wavelength in millimeters, $n$ is the number concentration of ice particles in inverse meters cubed (set to $1 \mathrm{~m}^{-3}$ herein), and the scattering amplitude elements, $S_{h h}$ and $S_{v v}$, have units of millimeters and are for forward-scattered radiation. For particles small relative to the wavelength, the real and imaginary parts of the forward-scattering amplitudes have the same magnitude as those of the backscattering amplitudes at each polarization (van de Hulst 1981). The underestimates of $K_{\mathrm{DP}}$ are similar to those for $Z_{\mathrm{DR}}$ because both variables depend on differences in the near-field interactions at horizontal and vertical polarization, through $S_{h h}$ and $S_{v v}$.

The maximum errors in $K_{\mathrm{DP}}$ are $\sim 0.6$ orders of magnitude (Fig. 5). Because $K_{\mathrm{DP}}$ depends on the number concentration of ice particles in the radar sampling volume, the value of these errors in degrees per kilometer will increase as the ice particle concentration increases.
For ice particle populations containing mostly smaller, higher-density $\left(>400 \mathrm{~kg} \mathrm{~m}^{-3}\right)$ planar ice crystals, the total $K_{\mathrm{DP}}$ error will be less than 0.4 orders of magnitude.

\section{b. Features of the internal fields of branched planar crystals}

To further illustrate the impact of ice particle structure on the scattering, we examine the internal electric field changes of individual dipoles within branched planar crystals (following Lu et al. 2013) and solid-ice plates of the same size and aspect ratio. We define these internal field changes as how much the internal field amplitude of a dipole (output by the DDA code) increases or decreases relative to the internal field amplitude of an isolated dipole of the same size and dielectric constant as those composing the branched planar crystals. Thus, the internal field change is a measure of how much the nearfield interactions of neighboring dipoles enhance or reduce the strength of the internal electric field at the location of a given dipole. For illustrative purposes, the internal field enhancements we show in Fig. 6 are for dipoles contained in the horizontal plane within the center of each particle.

At horizontal polarization, the plate has a nearly homogeneous internal field enhancement of $60 \%-70 \%$ (Fig. 6b). The only exceptions are along the particle 


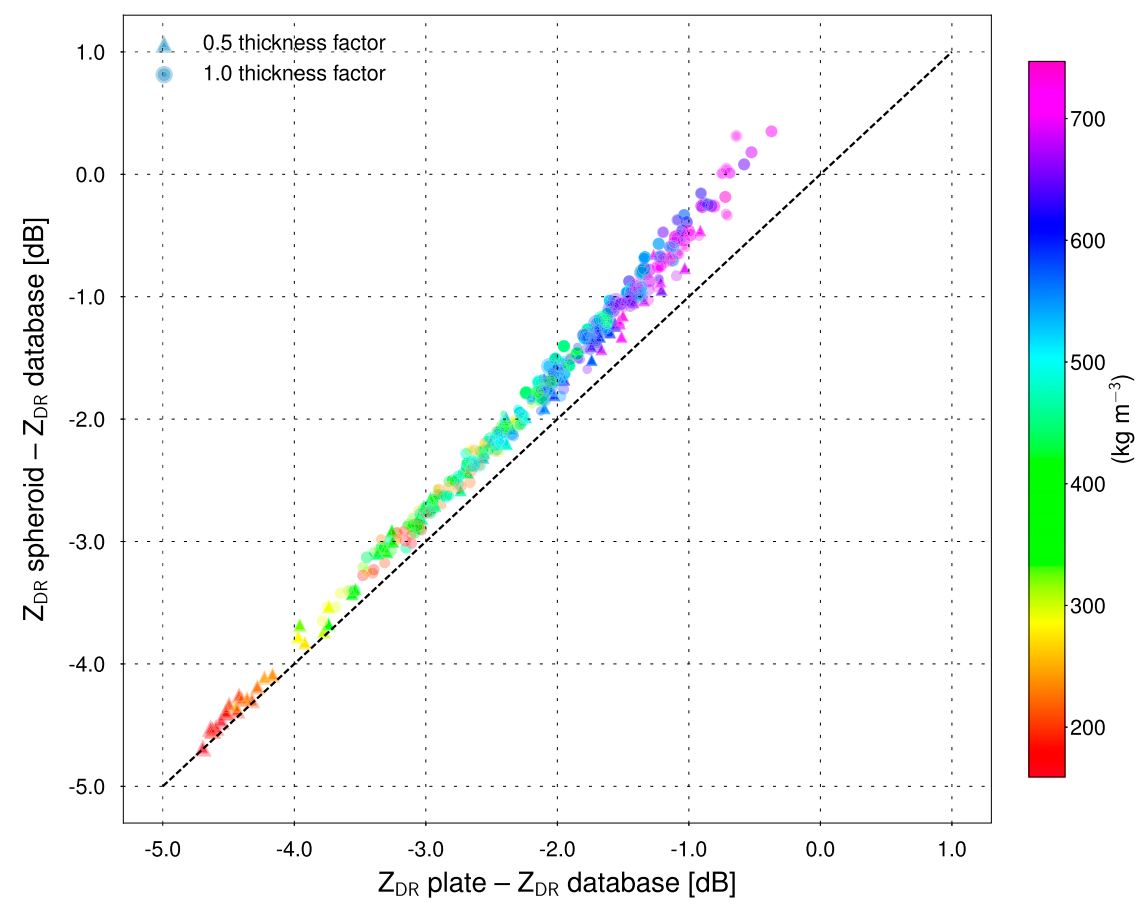

FIG. 4. As in Fig. 1, but of the $Z_{\mathrm{DR}}$ error for homogeneous, reduced-density spheroids vs $Z_{\mathrm{DR}}$ error of homogeneous, reduced-density plates.

edges, where internal field enhancements rapidly decrease to $\sim 20 \%$ near the edge oriented $60^{\circ}$ from the positive $x$ direction and increase to $\sim 75 \%$ at the edge oriented along the $x$ direction. In contrast, the internal field enhancements within the dendrite have much greater spatial variability (Fig. 6a). The largest enhancements $(>80 \%)$, greater than any within the plate, are found in the branches and subbranches oriented along the positive $x$ direction. These enhancements are larger than those found in the majority of plate dipoles because of the smaller number of dipole neighbors in the $y$ direction that would reduce the internal field enhancement of their neighbors. Internal field enhancements within the branches $60^{\circ}$ from horizontal have smaller internal field enhancements; slight reductions in the internal field magnitude are found within the edges of the subbranches pointing along the $y$ direction. These reductions are caused by the relatively fewer dipole neighbors in the $x$ direction than in the $y$ and $z$ directions within these regions of the particle.

Similar to the internal field at horizontal polarization, the plate has relatively homogeneous internal field reductions for vertically polarized radiation; the greatest reductions of $45 \%$ occur away from the edges of the plate. The internal field reductions are somewhat reduced to $\sim 30 \%$ along the plate edges. In contrast to the horizontal polarization results, the internal field reductions for vertically polarized radiation in the dendrite are smaller than the largest internal field reductions within the plate. These lesser reductions in the dendrite are caused by the relatively fewer neighboring dipoles in the $x$ and $y$ directions that if present, would further reduce the internal field magnitude. The dependence of the internal field changes on the orientation of the branches relative to the $x$ direction is also no longer present because of the electric field at vertical polarization always being normal to the orientations of the dendrite branches and subbranches.

\section{c. Effects of resonance}

Because the Lu et al. (2016) database does not include calculations at wavelengths longer than $\mathrm{X}$ band, and we seek to explore the errors of homogeneous reduceddensity plates at multiple weather-radar wavelengths, we perform additional scattering calculations (see Table 1 for further details) at frequencies of S, C, and X band, as well as at $\mathrm{Ku}-\mathrm{W}$ bands to explore the resonance behavior. We calculate the scattering at these frequencies for the lowest- and highest-mass branched planar crystals in the PSU database with maximum dimension of $5.0 \mathrm{~mm}$ and thickness factor of 1.0: the lowest-mass particle has sparsely distributed branches and subbranches and the highest-mass particle has densely distributed branches and subbranches, herein referred to as sparse dendrite and dense dendrite, respectively (Fig. 7). We use these dimensions because 


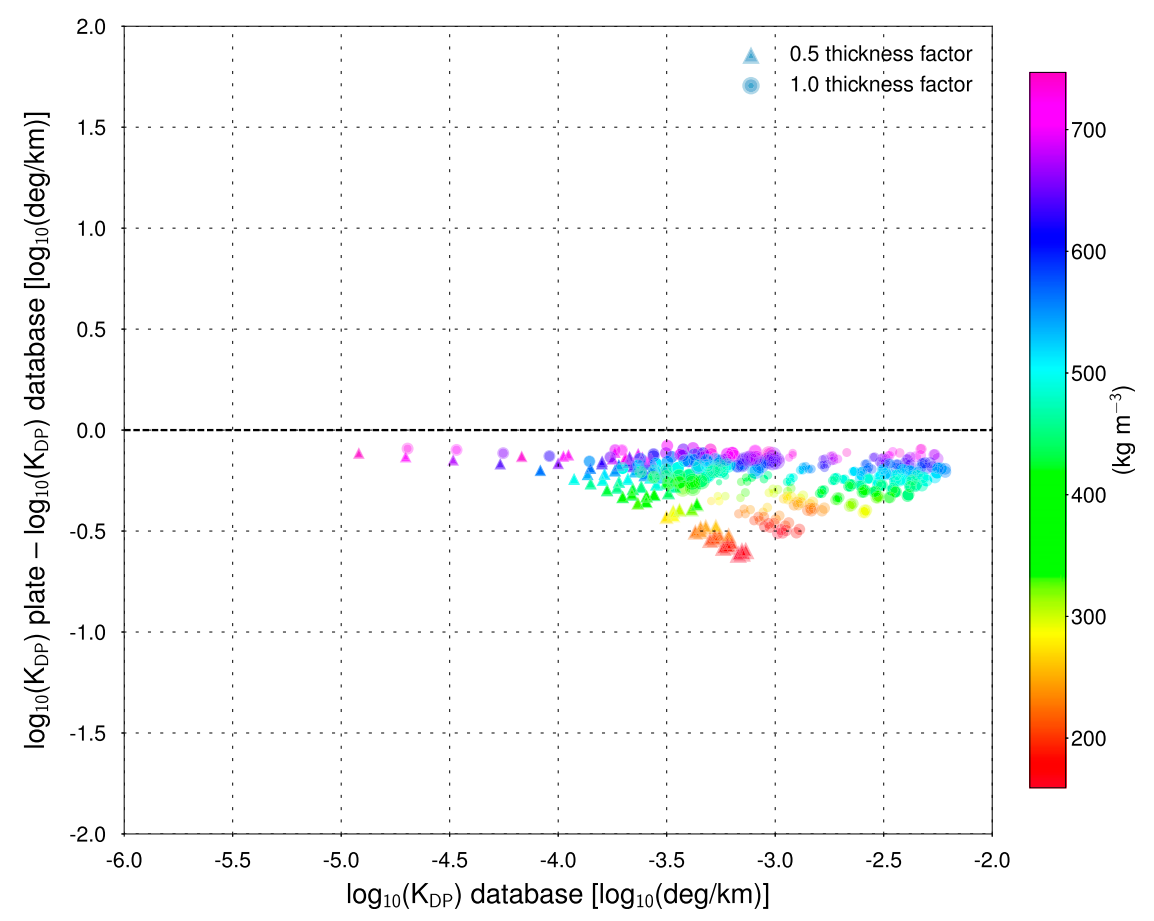

FIG. 5. As in Fig. 1, but for $K_{\mathrm{DP}}$.

these particles within the database contain the largest range in $\rho_{\mathrm{hp}}$. The 1.0-thickness-factor particles also exhibit larger resonance effects than the 0.5-thicknessfactor particles because of their larger mass. Thus, these particles will exhibit relatively large resonance effects; the resonance effects for the majority of observed branched planar crystals will be smaller.

We perform calculations for these dendrites and for corresponding homogeneous plates (as illustrated in section $3 b$ ) to assess the errors in using homogeneous, reduced-density particles to represent the scattering of branched planar crystals at all radar wavelengths. DDA is used to calculate the scattering amplitudes of all of the particles, and we use these to calculate the equivalent radar reflectivity factor (herein "reflectivity") at horizontal polarization $\left(Z_{H}\right)$, reflectivity at vertical polarization $\left(Z_{V}\right)$, and $Z_{\mathrm{DR}}$. We examine reflectivities instead of backscatter cross sections because, for wavelengths large relative to the particle size, $Z_{H}$ and $Z_{V}$ are independent on the wavelength of the incident radiation, and deviations in $Z_{H}$ and $Z_{V}$ as the wavelength decreases indicate resonance effects. The values of $Z_{H}$ and $Z_{V}$ are calculated from $\sigma_{h}$ and $\sigma_{\tau}$ with (Doviak and Zrnić 1993)

$$
Z_{H, V}=10 \times \log _{10}\left(\frac{n \lambda^{4}}{\pi^{5}\left|K_{w}\right|^{2}} \sigma_{h, v}\right),
$$

where $\left|K_{w}\right|^{2}=0.93, \lambda$ has units of millimeters, $n$ is $1 \mathrm{~m}^{-3}$, and $\sigma_{h, v}$ has units of millimeters squared.

At long wavelengths (S-Ku band), values of $Z_{H}$ and $Z_{V}$ for all particles increase by $\sim 1 \mathrm{~dB}$ from $\mathrm{Ku}$ to $\mathrm{X}$ band, increase by $<1 \mathrm{~dB}$ from $\mathrm{X}$ to $\mathrm{C}$ band, and remain nearly constant between $\mathrm{C}$ and $\mathrm{S}$ band (Fig. 8a). As observed in the $\mathrm{X}$-band comparisons in the previous section, there are significant errors (differences between homogeneous plates and the corresponding branched planar crystals) in $Z_{H}$ and $Z_{V}$ at these longer wavelengths where resonance effects are negligible; the sparse plate has a larger $Z_{H}$ error magnitude and a smaller $Z_{V}$ error magnitude compared to the dense plate (Fig. 8). As a result, the error in $Z_{\mathrm{DR}}$ for the low- $\rho_{\mathrm{hp}}$ plate is greater than the error in $Z_{\mathrm{DR}}$ for the high- $\rho_{\mathrm{hp}}$ plate. The dense dendrite has a $Z_{\mathrm{DR}}$ that is about $1 \mathrm{~dB}$ greater

TABLE 1. Description of the two branched planar crystals we use to illustrate the scattering behavior of branched planar crystals at different wavelengths, where $h$ is the particle thickness, $d$ is the particle maximum dimension in the horizontal plane, $b$ is the branch width in the direction normal to the branch orientation, $\rho_{\mathrm{hp}}$ is the homogeneous plate effective density, and $\lambda$ is the wavelength.

\begin{tabular}{ccccccc}
\hline \hline Name & Aspect ratio $(h / d)$ & $d(\mathrm{~mm})$ & $b(\mathrm{~mm})$ & $\rho_{\mathrm{hp}}\left(\mathrm{kg} \mathrm{m}^{-3}\right)$ & No. of dipoles & $\lambda(\mathrm{mm})$ \\
\hline Sparse dendrite & 0.012 & 5.0 & 0.097 & 177 & 663160 & $3.19,8.4,22.4,31.9,55.0,100.0$ \\
Dense dendrite & 0.012 & 5.0 & 0.31 & 673 & 2525080 & $3.19,8.4,22.4,31.9,55.0,100.0$ \\
\hline
\end{tabular}


a) Dendrite, $X$ polarization
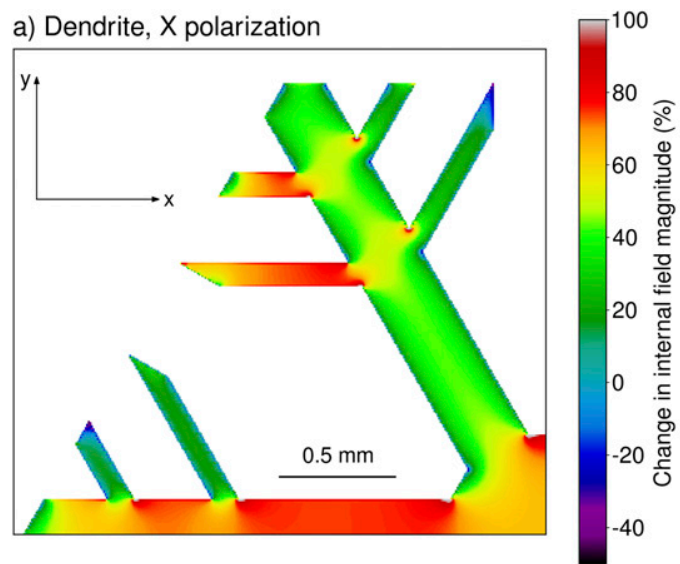

c) Dendrite, Z polarization
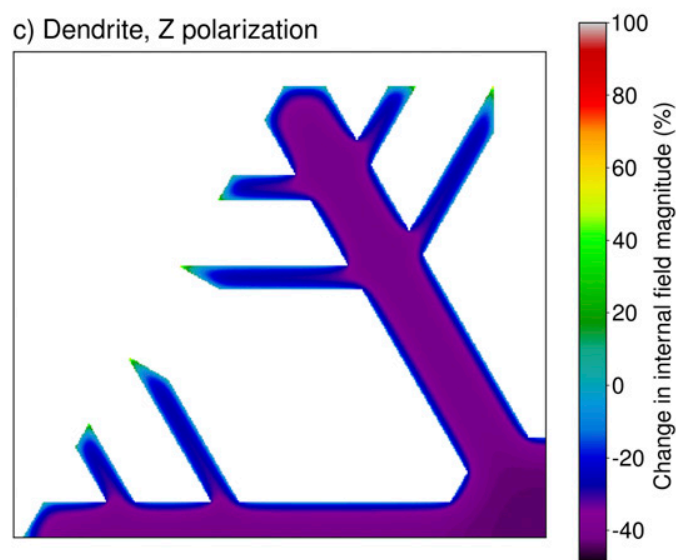

b) Plate, $X$ polarization

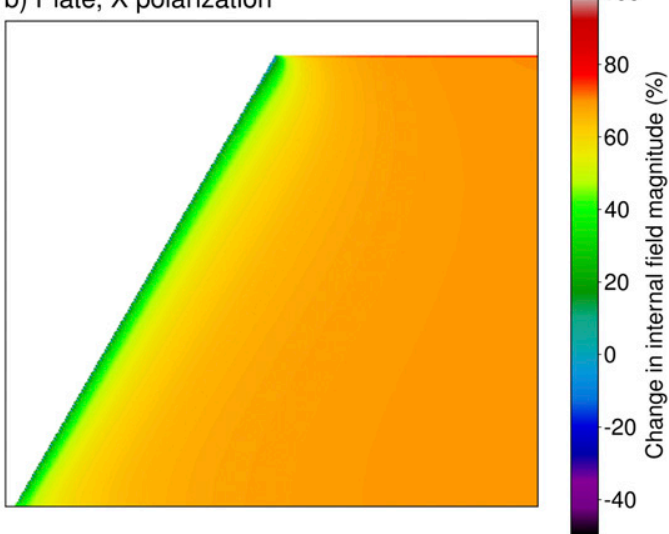

d) Plate, Z polarization

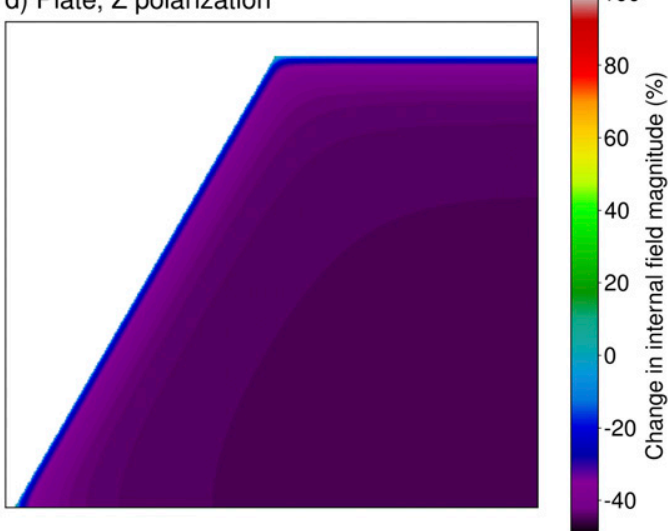

FIG. 6. Scatterplot of dipole locations shaded by the percentage enhancement (positive values) or reduction (negative values) of internal electric field amplitude relative to an isolated solid-ice dipole of the same sizes as the dipoles within the particles. The dipole locations are plotted for the upper-left quadrants of the branched planar crystal. The internal field strength changes are plotted for (a) horizontally polarized ( $x$ direction) radiation incident to a dendrite, (b) horizontally polarized radiation incident to a plate, $(\mathrm{c})$ vertically polarized $(z$ direction) radiation incident to a dendrite, and (d) vertically polarized radiation incident to a plate. The $x$ and $y$ directions (the $z$ direction is off the page) as well as a length scale of $0.5 \mathrm{~mm}$ are plotted and labeled in (a).

than the corresponding dense plate; the sparse dendrite has a $Z_{\mathrm{DR}} \sim 3 \mathrm{~dB}$ greater than the corresponding sparse plate (Fig. 8c).

Resonance effects are noticeable at wavelengths $<22.4 \mathrm{~mm}$ (Ku band). Decreases in $Z_{H}$ from $\mathrm{Ku}$ to $\mathrm{Ka}$ band range from 17 to $23 \mathrm{~dB}$, with the smallest change occurring for the dense dendrite. From $\mathrm{Ka}$ to $\mathrm{W}$ band, $Z_{H}$ decreases by $3-4 \mathrm{~dB}$ for the homogeneous plates and increases by 3 and $17 \mathrm{~dB}$ for the dense and sparse dendrites, respectively (Fig. 8a). A similar behavior is observed for the $Z_{V}$ results, with values decreasing by $19-28 \mathrm{~dB}$ from $\mathrm{Ku}$ to $\mathrm{Ka}$ band; from Ka to $\mathrm{W}$ band, $Z_{V}$ decreases by $2-3 \mathrm{~dB}$ for the homogeneous plates and increases by 2 and $20 \mathrm{~dB}$ for the dense and sparse dendrites, respectively (Fig. 8b).

Errors in $Z_{\mathrm{DR}}$ at shorter wavelengths, due to underestimates of the near-field interactions, are amplified by resonance effects. The error in $Z_{\mathrm{DR}}$ decreases from $-3 \mathrm{~dB}$ at $\mathrm{X}$ band to $\sim-7.5 \mathrm{~dB}$ at $\mathrm{Ka}$ band for the sparse particle, and it decreases from $-1 \mathrm{~dB}$ at $\mathrm{X}$ band to $\sim-1.5 \mathrm{~dB}$ at Ka band for the dense particle. From Ka to $\mathrm{W}$ band, the $Z_{\mathrm{DR}}$ error increases to $\sim-5 \mathrm{~dB}$ for the sparse particle and decreases to $\sim-2.5 \mathrm{~dB}$ for the dense particle (Fig. 8c). Some of the error occurs because nearfield interactions between dipoles in the branched planar crystals are stronger than those in homogeneous plates. Thus the phase-diverse sum of scattered waves from dipoles within branched planar crystals will deviate more from small-particle scattering than that from homogeneous plates. However, inhomogeneous distributions of mass within the branched planar crystals may also lead to less-predictable resonance effects, where individual structures (e.g., branches, subbranches, and the core) may have dipole oscillations that are more or 
a) sparse dendrite

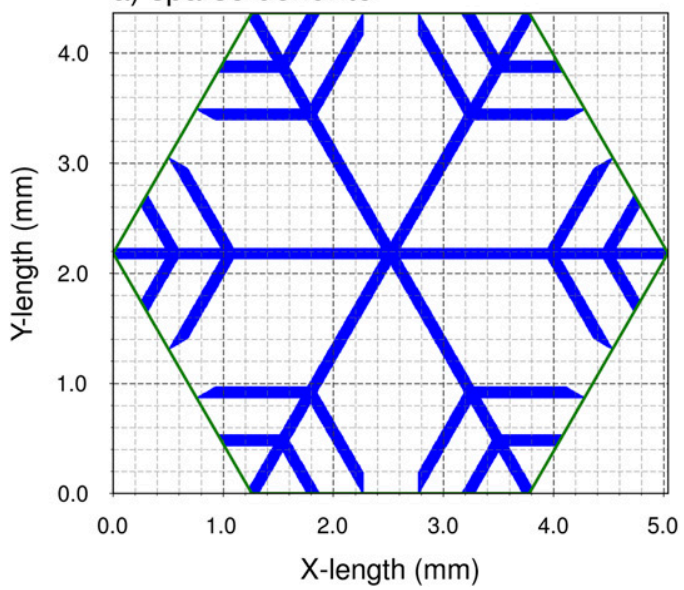

b) dense dendrite

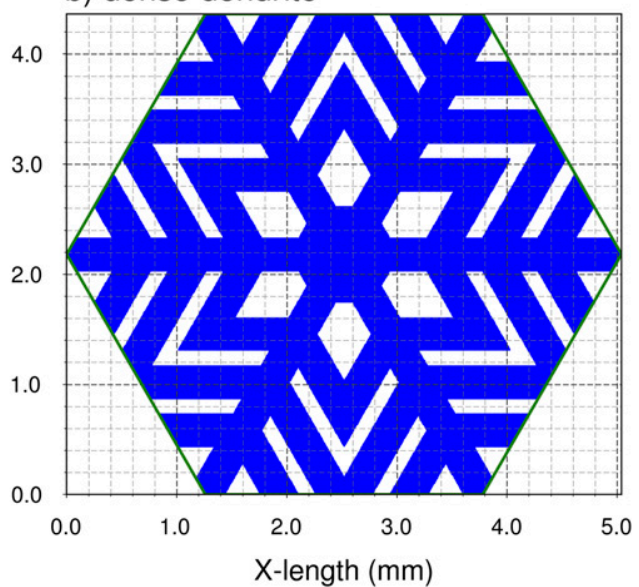

FIG. 7. Top view of the (a) sparse branched planar crystal and (b) dense branched planar crystal. The blue region indicates areas where dipoles are assigned and the green line along the peripheries of the crystals indicates the bounding hexagon of each shape.

less in-phase than the dipole oscillations within corresponding homogeneous plates. Results for 5.6-mm particles (not shown) reveal a large peak in $Z_{\mathrm{DR}}$ of $\sim 10 \mathrm{~dB}$ for the sparse plate at Ka band, further illustrating the extreme sensitivity resonance effects have to the dimensions relative to the wavelength of low-aspect-ratio platelike ice particles.

\section{Discussion and conclusions}

Characterizing the scattering of pristine ice particles is necessary to interpret polarimetric radar observations of these particles. Consistent polarimetric signatures near $-15^{\circ} \mathrm{C}$ in stratiform precipitation (e.g., Kennedy and
Rutledge 2011; Andrić et al. 2013; Schrom et al. 2015) have prompted recent interest in determining whether this signature reflects the rapid depositional growth of branched planar crystals. Many previous works represent these branched planar crystals as homogeneous, reduceddensity spheroids with bulk density-size and aspect ratiosize empirical relationships, and use the scattering of these spheroids to make inferences about the degree of vapor depositional growth, aggregation, and riming indicated by the radar observations.

Our results presented herein indicate that there are significant differences in the polarimetric radar quantities of branched planar ice crystals and homogeneous, reduced-density plates with the same masses, aspect
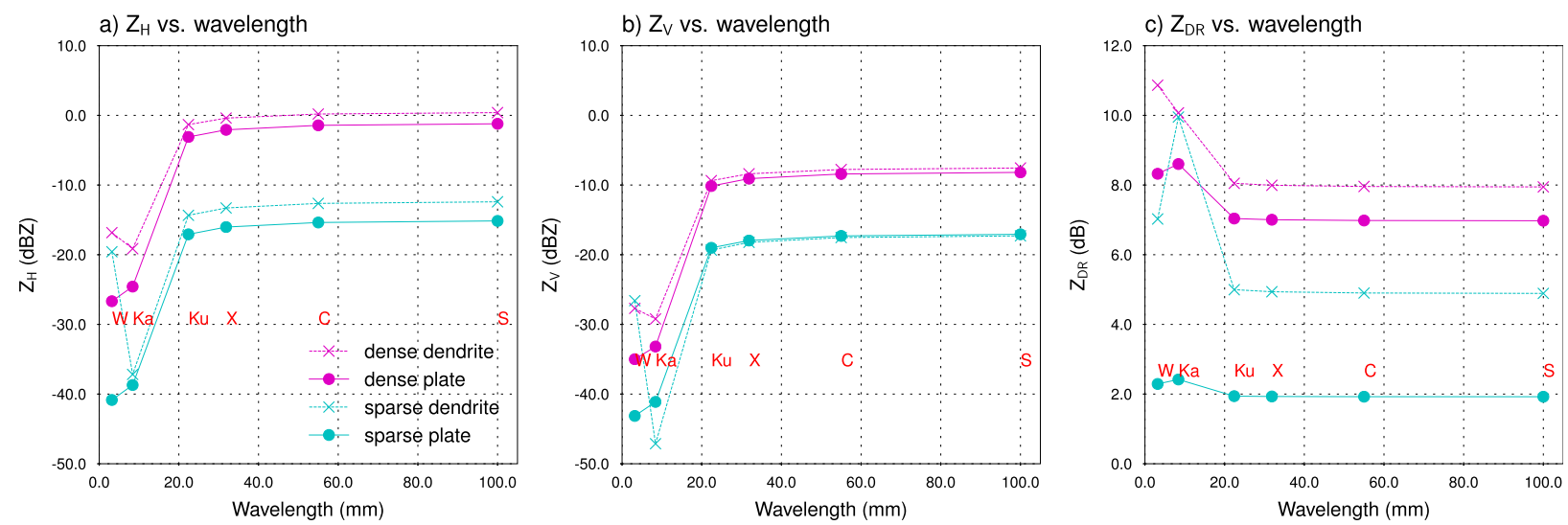

FIG. 8. Calculations of (a) $Z_{H}$, (b) $Z_{V}$, and (c) $Z_{\mathrm{DR}}$ as a function of wavelength for the particles described in Table 1 . The magenta dashed line with cross markers indicates calculations for the dense dendrite, the magenta solid line with circular markers indicates calculations for the homogeneous plate with the same density as the dense dendrite, the cyan dashed line with cross markers indicates calculations for the sparse dendrite, and the cyan solid line with circular markers indicates calculations for the homogeneous plate with the same density as the sparse dendrite. The red letters labeled in each plot indicate the name of the frequency band corresponding to the wavelength of the calculations. 
ratios, and maximum dimensions. Homogeneous plates generally underestimate $\sigma_{h}$ more than $\sigma_{v}$, with these errors having a large dependence on the detailed structure of the branched planar crystal. The magnitude of the $Z_{\mathrm{DR}}$ and $K_{\mathrm{DP}}$ errors generally increases as the effective density and aspect ratio decrease, though the shape and dimensions of the core, branches, and subbranches also have substantial impacts on the error. These errors occur at all wavelengths, with the X-band comparisons of the branched planar crystals from the $\mathrm{Lu}$ et al. (2016) database indicating that poor representations of the near-field interactions within branched planar crystals by homogeneous plates can lead to underestimates of $Z_{\mathrm{DR}}$ up to $4.5 \mathrm{~dB}$. Because resonance effects are minimal from $X$ to $S$ band for crystals in the database, we expect these errors in $Z_{\mathrm{DR}}$ to be similar at $\mathrm{C}$ and $\mathrm{S}$ band. Canting (not considered in this work) will cause the $Z_{\mathrm{DR}}$ of homogeneous particles to decrease less than the $Z_{\mathrm{DR}}$ of branched planar crystals, reducing the magnitude of the $Z_{\mathrm{DR}}$ errors somewhat.

Errors in $Z_{H}, Z_{V}, Z_{\mathrm{DR}}$, and $K_{\mathrm{DP}}$ that occur for particles that are small relative to the wavelength are caused by underestimates in the strength of the nearfield interactions between horizontally aligned dipoles in the core, branches, and subbranches. These underestimates occur primarily because the magnitudes of electric dipole near fields decrease with $r^{-3}$ and dipoles within branched planar crystals are closely packed within thin branches, subbranches, and core regions. Because homogeneous particles are effectively composed of uniformly distributed solid-ice dipoles, distances between neighboring dipoles are maximized and the thus the near-field interactions are minimized. As suggested by theory, the differences in near-field interaction strength between branched planar and homogeneous-plate crystals should decrease as the aspect ratio increases, potentially explaining the small differences in scattering between the branched planar and homogeneous-plate crystals with aspect ratio of 0.1 from Westbrook (2014).

At wavelengths where these resonance effects occur, the errors in representing branched planar crystals with homogeneous plates are generally amplified. This amplification of the error occurs because the stronger nearfield interactions of the branched planar crystals allow for stronger scattered waves from individual dipoles relative to the equivalent, reduced-density homogeneous plates with weaker near-field interactions. The out-of-phase sum of stronger scattered fields leads to greater deviations from small-particle scattering. The detailed ice crystal structure also has a large impact on resonance effects, with less predictable changes in $Z_{H}$, $Z_{V}$, and $Z_{\mathrm{DR}}$ with decreasing radar wavelength. Because of the generally different resonance behavior between homogeneous plates and branched planar crystals, the magnitude of the error at a particular wavelength may occasionally decrease below the long-wavelength error.

Numerous studies cite observations of large $Z_{\mathrm{DR}}$ as indicative of high-density plates because their interpretations are informed by the scattering of homogeneous spheroids (e.g., Kennedy and Rutledge 2011; Andrić et al. 2013; Schrom et al. 2015). However, X-band observations from Oue et al. (2016) show $Z_{\mathrm{DR}}>$ $7 \mathrm{~dB}$ through the entire depth of an Arctic mixed-phase cloud, coincident with photographic evidence of dendrites at the surface. Comparable values of $Z_{\mathrm{DR}}$ have also been observed by the authors in winter precipitation in the northeastern United States with operational S-band Weather Surveillance Radar-1988 Doppler (WSR-88D) data. If homogeneous, reduceddensity spheroids are used to represent the scattering of these ice particles, only low-aspect-ratio, high-effectivedensity particles (i.e., solid plate crystals) will be able to reproduce the observed $Z_{\mathrm{DR}}$ values. Homogeneous, reduced-density spheroids with very low aspect ratios and low effective densities (representative of thin, branched planar crystals) will not be able to produce such high $Z_{\mathrm{DR}}$ values. However, scattering calculations from the Lu et al. (2016) database of sparse, low-aspectratio branched planar crystals indicate that these particles may indeed be able to produce the observed $Z_{\mathrm{DR}}$. Therefore, suggestions that $Z_{\mathrm{DR}}$ observations can be used to discriminate between plates and dendrites may have to be revisited. These results also increase the uncertainty in the retrieval of aspect ratios from $Z_{\mathrm{DR}}$ measurements, since dendrites may have much lower aspect ratios but comparable $Z_{\mathrm{DR}}$ to plates.

Observations of $K_{\mathrm{DP}}$ scaling with the radar wavelength (Bechini et al. 2013) indicate that pristine ice crystals scatter in the small particle regime. These observations are consistent with our results for branched planar crystals at $\mathrm{X}$ band. The $K_{\mathrm{DP}}$ errors we show are, similar to $Z_{\mathrm{DR}}$, a result of the homogeneous, reduced-density particles underestimating the near-field interactions between dipoles in the core, branches, and subbranches of dendrites. The underestimates of $K_{\mathrm{DP}}$ by homogeneous, reduced-density particles suggest that smaller number concentrations than previously thought may be responsible for the enhanced $K_{\mathrm{DP}}$ observations near $-15^{\circ} \mathrm{C}$ (e.g., Andrić et al. 2013; Moisseev et al. 2015).

These results also have several implications for comparisons of microphysical model output with polarimetric radar observations. The large spread in calculations of the polarimetric radar variables, particularly $Z_{\mathrm{DR}}$, for different realizations of ice particles within a narrow range of sizes, aspect ratios, and effective densities 
suggests that care must be taken in developing the forward model to simulate these variables from model output. Several recently developed microphysical schemes allow for the natural evolution of ice particle properties (e.g., mass, size, and/or aspect ratio) during vapor deposition, aggregation, and riming (e.g., Harrington et al. 2013; Jensen and Harrington 2015; Morrison and Milbrandt 2015). Directly mapping the simulated particle properties to homogeneous, reduced-density spheroids and using these particles to calculate the radar variables (e.g., Ryzhkov et al. 2011; Andrić et al. 2013; Sulia and Kumjian 2017) will lead to erroneous comparisons with polarimetric radar observations. It is therefore necessary to map these simulated ice particle properties to scattering calculations that correspond to realistic particle shapes before conducting these comparisons.

Our results clearly indicate that homogeneous, reduced-density particles (i.e., plates and spheroids) are insufficient to capture the scattering of branched planar ice crystals with very low aspect ratios and effective density $<400 \mathrm{~kg} \mathrm{~m}^{-3}$ at all radar wavelengths. To accurately compute the radar variables, we therefore need additional quantities beyond effective density to describe the distribution of mass within branched planar crystals. These additional quantities may include the number of branches and subbranches and the relative lengths of the subbranches. Using this additional information about the ice particle structure to simulate the polarimetric radar variables with analytical functions is often desirable. One such approach outlined in Westbrook (2014) is to compute the scattering amplitude elements of branched planar crystals with DDA and derive empirical relationships for these elements with respect to size, aspect ratio, and the number of branches and subbranches and the relative lengths of the subbranches. Another approach is to use two-layer spheroids, derived using the ice particle structure information, to estimate the scattering of branched planar crystals. Preliminary results using this method with unique effective densities for the branch and core regions show reductions in the error of $Z_{H}, Z_{V}$, and $Z_{\mathrm{DR}}$ (not shown). However, before further exploring these methods for approximating the scattering of branched planar crystals, we may first need to better quantify how the relationship between the core, branches, and subbranches impacts the scattering of branched planar ice crystals.

Acknowledgments. Funding for the authors comes from DOE Grant DE-SC0013953. We thank Hans Verlinde, Eugene Clothiaux, and Kultegin Aydin for contributing to insightful discussions about this project. We thank the reviewers for their constructive feedback that significantly improved the quality of the manuscript.

\section{REFERENCES}

Andrić, J., M. R. Kumjian, D. S. Zrnić, J. M. Straka, and V. M. Melnikov, 2013: Polarimetric signatures above the melting layer in winter storms: An observational and modeling study. J. Appl. Meteor. Climatol., 52, 682-700, https://doi.org/ 10.1175/JAMC-D-12-028.1.

Auer, A. H., and D. L. Veal, 1970: The dimension of ice crystals in natural clouds. J. Atmos. Sci., 27, 919-926, https://doi.org/ 10.1175/1520-0469(1970)027<0919:TDOICI > 2.0.CO;2.

Aydin, K., J. Verlinde, E. E. Clothiaux, Y. Lu, Z. Jiang, and G. Botta, 2016: Polarimetric scattering database for non-spherical ice particles at microwave wavelengths. Atmospheric Radiation Measurement (ARM) Climate Research Facility Data Archive, accessed 8 March 2017, https://doi.org/10.5439/1258029.

Bailey, M. P., and J. Hallett, 2009: A comprehensive habit diagram for atmospheric ice crystals: Confirmation from the laboratory, AIRS II, and other field studies. J. Atmos. Sci., 66, 28882899, https://doi.org/10.1175/2009JAS2883.1.

Bechini, R., L. Baldini, and V. Chandrasekar, 2013: Polarimetric radar observations in the ice region of precipitating clouds at C-band and X-band radar frequencies. J. Appl. Meteor. Climatol., 52, 1147-1169, https://doi.org/10.1175/JAMC-D-12-055.1.

Bohren, C. F., and D. R. Huffman, 1983: Absorption and Scattering of Light by Small Particles. 1st ed. John Wiley and Sons, 530 pp.

Botta, G., K. Aydin, J. Verlinde, A. E. Avramov, A. S. Ackerman, A. M. Fridlind, G. M. McFarquhar, and M. Wolde, 2011: Millimeter wave scattering from ice crystals and their aggregates: Comparing cloud model simulations with X- and Kaband radar measurements. J. Geophys. Res., 116, D00T04, https://doi.org/10.1029/2011JD015909.

,$- \longrightarrow$, and -2013 : Variability in millimeter wave scattering properties of dendritic ice crystals. J. Quant. Spectrosc. Radiat. Transfer, 131, 105-114, https://doi.org/10.1016/ j.jqsrt.2013.05.009.

Bringi, V. N., and V. Chandrasekar, 2001: Polarimetric Doppler Weather Radar: Principles and Applications. 1st ed. Cambridge University Press, $636 \mathrm{pp}$.

Connolly, P. J., C. Emersic, and P. R. Field, 2012: A laboratory investigation into the aggregation efficiency of small ice crystals. Atmos. Chem. Phys., 12, 2055-2076, https://doi.org/ 10.5194/acp-12-2055-2012.

Doviak, R. J., and D. S. Zrnić, 1993: Doppler Radar and Weather Observations. 2nd ed. Academic Press, 562 pp.

Draine, B. T., and P. J. Flatau, 1994: Discrete-dipole approximation for scattering calculations. J. Opt. Soc. Amer., 11A, 14911499, https://doi.org/10.1364/JOSAA.11.001491.

Fukuta, N., and T. Takahashi, 1999: The growth of atmospheric ice crystals: A summary of findings in vertical supercooled cloud tunnel studies. J. Atmos. Sci., 56, 1963-1979, https://doi.org/ 10.1175/1520-0469(1999)056<1963:TGOAIC >2.0.CO;2.

Hall, M. P. M., J. W. F. Goddard, and S. M. Cherry, 1984: Identification of hydrometeors and other targets by dualpolarization radar. Radio Sci., 19, 132-140, https://doi.org/ 10.1029/RS019i001p00132.

Harrington, J. Y., K. Sulia, and H. Morrison, 2013: A method for adaptive habit prediction in bulk microphysical models. Part I: Theoretical development. J. Atmos. Sci., 70, 349-364, https:// doi.org/10.1175/JAS-D-12-040.1. 
Hashino, T., and G. J. Tripoli, 2007: The Spectral Ice Habit Prediction System (SHIPS). Part I: Model description and simulation of the vapor deposition process. J. Atmos. Sci., 64, 2210-2237, https://doi.org/10.1175/JAS3963.1.

Jackson, J. D., 1975: Classical Electrodynamics. 2nd ed. John Wiley and Sons, $848 \mathrm{pp}$

Jensen, A. A., and J. Y. Harrington, 2015: Modeling ice crystal aspect ratio evolution during riming: A single-particle growth model. J. Atmos. Sci., 72, 2569-2590, https://doi.org/10.1175/JAS-D-14-0297.1.

Kennedy, P. C., and S. A. Rutledge, 2011: S-band dual-polarization radar observations of winter storms. J. Appl. Meteor. Climatol., 50, 844-858, https://doi.org/10.1175/2010JAMC2558.1.

Kneifel, S., A. von Lerber, J. Tiira, D. Moisseev, P. Kollias, and J. Leinonen, 2015: Observed relations between snowfall microphysics and triple-frequency radar measurements. J. Geophys. Res. Atmos., 120, 6034-6055, https://doi.org/10.1002/2015JD023156.

Lemke, H. M., and M. Quante, 1999: Backscatter characteristics of nonspherical ice crystals: Assessing the potential of polarimetric radar measurements. J. Geophys. Res., 104, 31739 31 751, https://doi.org/10.1029/1999JD900490.

Lu, Y., E. E. Clothiaux, K. Aydin, G. Botta, and J. Verlinde, 2013: Modeling variability in dendritic ice crystal backscattering cross sections at millimeter wavelengths using a modified Rayleigh-Gans theory. J. Quant. Spectrosc. Radiat. Transfer, 131, 95-104, https://doi.org/10.1016/j.jqsrt.2013.05.008.

,,--- , and J. Verlinde, 2014: Estimating ice particle scattering properties using a modified Rayleigh-Gans approximation. J. Geophys. Res. Atmos., 119, 10 471-10484, https://doi.org/ 10.1002/2014JD021850.

, Z. Jiang, K. Aydin, J. Verlinde, E. E. Clothiaux, and G. Botta, 2016: A polarimetric scattering database for non-spherical ice particles at microwave wavelengths. Atmos. Meas. Tech., 9, 5119-5134, https://doi.org/10.5194/amt-9-5119-2016.

Matrosov, S. Y., R. F. Reinking, R. A. Kropfli, and B. W. Bartram, 1996: Estimation of ice hydrometeor types and shapes from radar polarization measurements. J. Atmos. Oceanic Technol., 13, 85-96, https://doi.org/10.1175/1520-0426(1996)013<0085: EOIHTA $>2.0 . \mathrm{CO} ; 2$.

Maxwell Garnett, J. C., 1904: Colours in metal glasses and in metallic films. Philos. Trans. Roy. Soc. London, 203A, 385-420, https://doi.org/10.1098/rsta.1904.0024.

Moisseev, D. N., S. Lautaportti, J. Tyynela, and S. Lim, 2015: Dualpolarization radar signatures in snowstorms: Role of snowflake aggregation. J. Geophys. Res. Atmos., 120, 12 644-12 655, https://doi.org/10.1002/2015JD023884.

Morrison, H., and J. A. Milbrandt, 2015: Parameterization of cloud microphysics based on the prediction of bulk ice particle properties. Part I: Scheme description and idealized tests. J. Atmos. Sci., 72, 287-311, https://doi.org/10.1175/JAS-D-14-0065.1.
Oue, M., M. Galletti, J. Verlinde, A. Ryzhkov, and Y. Lu, 2016: Use of X-band differential reflectivity measurements to study shallow Arctic mixed-phase clouds. J. Appl. Meteor. Climatol., 55, 403-424, https://doi.org/10.1175/JAMC-D-15-0168.1.

Ryzhkov, A. V., D. S. Zrnić, and B. A. Gordon, 1998: Polarimetric method for ice water content determination. J. Appl. Meteor., 37, 125-134, https://doi.org/10.1175/1520-0450(1998)037<0125: PMFIWC $>2.0$.CO;2.

—, M. Pinsky, A. Pokrovsky, and A. Khain, 2011: Polarimetric radar observation operator for a cloud model with spectral microphysics. J. Appl. Meteor. Climatol., 50, 873-894, https:// doi.org/10.1175/2010JAMC2363.1.

Schrom, R. S., M. R. Kumjian, and Y. Lu, 2015: Polarimetric radar signatures of dendritic growth zones within Colorado winter storms. J. Appl. Meteor. Climatol., 54, 2365-2388, https://doi.org/ 10.1175/JAMC-D-15-0004.1.

Shivola, A. H., 1989: Self-consistency aspects of dielectric mixing theories. IEEE Trans. Geosci. Remote Sens., 27, 403-415, https://doi.org/10.1109/36.29560.

Spek, A. L. J., C. M. H. Unal, D. N. Moisseev, H. W. J. Russchenberg, V. Chandrasekar, and Y. Dufournet, 2008: A new technique to categorize and retrieve the microphysical properties of ice particles above the melting layer using radar dual-polarization spectral analysis. J. Atmos. Oceanic Technol., 25, 482-497, https://doi.org/10.1175/ 2007JTECHA944.1.

Sulia, K. J., and M. R. Kumjian, 2017: Simulated polarimetric fields of ice vapor growth using the adaptive habit model. Part I: Large-eddy simulations. Mon. Wea. Rev., 145, 2281-2302, https://doi.org/10.1175/MWR-D-16-0061.1.

Thompson, E. J., S. A. Rutledge, B. Dolan, V. Chandrasekar, and B. L. Cheong, 2014: A dual-polarization radar hydrometeor classification algorithm for winter precipitation. J. Atmos. Oceanic Technol., 31, 1457-1481, https://doi.org/ 10.1175/JTECH-D-13-00119.1.

van de Hulst, H. C., 1981: Light Scattering by Small Particles. 1st ed. Dover, $481 \mathrm{pp}$.

Vivekanandan, J., V. N. Bringi, M. Hagen, and P. Meischner, 1994: Polarimetric radar studies of atmospheric ice particles. IEEE Trans. Geosci. Remote Sens., 32, 1-10, https://doi.org/10.1109/ 36.285183.

Westbrook, C. D., 2014: Rayleigh scattering by hexagonal ice crystals and the interpretation of dual-polarisation radar measurements. Quart. J. Roy. Meteor. Soc., 140B, 2090-2096, https://doi.org/10.1002/qj.2262.

Yurkin, M. A., and A. G. Hoekstra, 2011: The discrete-dipoleapproximation code ADDA: Capabilities and known limitations. J. Quant. Spectrosc. Radiat. Transfer, 112, 2234-2247, https://doi.org/10.1016/j.jqsrt.2011.01.031. 